\title{
Falcarindiol Isolated from Notopterygium incisum Inhibits the Quorum Sensing of Pseudomonas aeruginosa
}

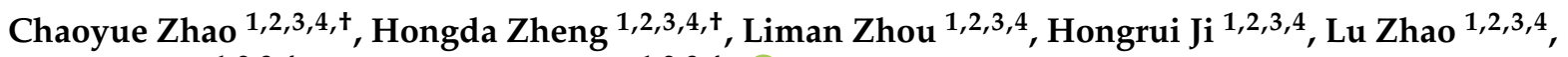 \\ Wengong $\mathrm{Yu}^{1,2,3,4}$ and Qianhong Gong $1,2,3,4, *$ (D) \\ 1 School of Medicine and Pharmacy, Ocean University of China, Yushan Road, Qingdao 266003, China; \\ zhaochaoyue2712@stu.ouc.edu.cn (C.Z.); hongdazh@yeah.net (H.Z.); zhouliman88@126.com (L.Z.); \\ jhr@stu.ouc.edu.cn (H.J.); zhaolu@stu.ouc.edu.cn (L.Z.); yuwg66@ouc.edu.cn (W.Y.) \\ 2 Laboratory for Marine Drugs and Bioproducts, Qingdao National Laboratory for Marine Science and \\ Technology, 1 Wenhai Road, Qingdao 266237, China \\ 3 Provincial Key Laboratory of Glycoscience and Glycotechnology, Ocean University of China, Yushan Road, \\ Qingdao 266003, China \\ 4 Key Laboratory of Marine Drugs, Chinese Ministry of Education, School of Medicine and Pharmacy, \\ Ocean University of China, 5 Yushan Road, Qingdao 266003, China \\ * Correspondence: gongqh@ouc.edu.cn \\ + These authors contributed equally to this work.
}

Citation: Zhao, C.; Zheng, H.; Zhou, L.; Ji, H.; Zhao, L.; Yu, W.; Gong, Q.

Falcarindiol Isolated from

Notopterygium incisum Inhibits the Quorum Sensing of Pseudomonas aeruginosa. Molecules 2021, 26, 5896. https://doi.org/10.3390/

molecules26195896

Academic Editor: Herman O. Sintim

Received: 20 July 2021

Accepted: 20 September 2021

Published: 29 September 2021

Publisher's Note: MDPI stays neutral with regard to jurisdictional claims in published maps and institutional affiliations.

Copyright: (c) 2021 by the authors. Licensee MDPI, Basel, Switzerland. This article is an open access article distributed under the terms and conditions of the Creative Commons Attribution (CC BY) license (https:/ / creativecommons.org/licenses/by/ $4.0 /)$.

\begin{abstract}
Quorum sensing (QS) is employed by the opportunistic pathogen Pseudomonas aeruginosa to regulate physiological behaviors and virulence. QS inhibitors (QSIs) are potential anti-virulence agents for the therapy of P. aeruginosa infection. During the screening for QSIs from Chinese herbal medicines, falcarindiol (the major constituent of Notopterygium incisum) exhibited QS inhibitory activity. The subinhibitory concentration of falcarindiol exerted significant inhibitory effects on the formation of biofilm and the production of virulence factors such as elastase, pyocyanin, and rhamnolipid. The mRNA expression of QS-related genes (lasB, phzH, rhlA, lasI, rhlI, pqsA, and $r h l R$ ) was downregulated by falcarindiol while that of $l a s R$ was not affected by falcarindiol. The transcriptional activation of the lasI promoter was inhibited by falcarindiol in the P. aeruginosa QSISlasI selector. Further experiments confirmed that falcarindiol inhibited the las system using the reporter strain Escherichia coli MG4/pKDT17. Electrophoretic mobility shift assay (EMSA) showed that falcarindiol inhibited the binding of the transcription factor LasR and the lasI promoter region. Molecular docking showed that falcarindiol interacted with the Tyr47 residue, leading to LasR instability. The decrease of LasR-mediated transcriptional activation was responsible for the reduction of downstream gene expression, which further inhibited virulence production. The inhibition mechanism of falcarindiol to LasR provides a theoretical basis for its medicinal application.
\end{abstract}

Keywords: falcarindiol; quorum sensing; Pseudomonas aeruginosa; Notopterygium incisum; LasR

\section{Introduction}

Pseudomonas aeruginosa is one of the most intractable opportunistic pathogens, and can cause acute and chronic human infections. Infections with P. aeruginosa are difficult to treat with antibiotics [1]. Antibiotics put enormous pressure on bacteria and lead to the emergence of multidrug-resistant strains [2]. Anti-virulence therapy is a novel anti-infection therapeutic strategy and could exert less selective pressure on bacteria [3]. Virulence in $P$. aeruginosa is mostly regulated by quorum sensing (QS), a complex network controlled by signal molecules. Therefore, quorum sensing inhibitors (QSIs) could be promising anti-virulence agents to treat $P$. aeruginosa infection [4].

QS regulates bacterial population behavior by secreting and detecting signal molecules. Three interwoven QS circuits are used by P. aeruginosa, including two acyl-homoserine lactone (AHL) systems (the las system and the $r h l$ system) and the PqsR-controlled pqs system [5]. The las and $r h l$ systems control the expression of more than $6 \%$ of the $P$. aeruginosa 
genome [6]. LasR works at the top of the regulatory hierarchy of the QS network [7,8]. LasI is the enzyme that synthesizes the signal molecular 3-oxo-dodecanoyl (3-oxo-C12)-HSL [9]. The receptor LasR binds with 3-oxo-C12-HSL and activates the expression of multiple genes, including lasI, rhlI, pqs A, lasB, rhlR, and pqsR, etc. [10]. Butyryl-HSL (C4-HSL) and alkyl quinolone signaling molecules activate and bind with the receptors RhlR and PqsR, respectively. RhlR inhibits the expression of $p q s R$ and $p q s A B C D$ while PqsR activates that of rhlRI.

Inhibiting the QS response is one of the strategies plants have evolved to deal with bacterial infections [11]. Many natural extracts have inhibitory activities on the quorum sensing of $P$. aeruginosa. The extract of Lavandula coronopifolia can inhibit the biofilm formation of $P$. aeruginosa. Methanol extract from Salix tetrasperma stem bark can inhibit the swimming motility, swarming motility, and protease production of $P$. aeruginosa [12,13]. QSIs such as zingerone, baicalin, and naringenin have been found in natural extracts against the common pathogen P. aeruginosa, which can infect both humans and plants [14-16]. Our research identified the polyacetylenic compound falcarindiol from Notopterygium incisum as having QS inhibitory activity. The effect of falcarindiol on the virulence of $P$. aeruginosa and the mechanisms of anti-QS activity were further evaluated.

\section{Results}

\subsection{Screening and Identification of Falcaridiol}

To screen quorum-sensing inhibitors, we constructed the PQSI selector strain (P. aeruginosa PAO1 harboring a PpqsA-sacB plasmid). $S a c B$ is the sucrose lethal gene. The growth of the selector strain was inhibited in the presence of sucrose and PQS. QSIs rescued the bacteria by inhibiting the transcription of the $p q s A$ promoter, and the OD600 nm value of the PQSI culture was increased. Because the $p q s A$ promoter is activated by PqsR and LasR, the PQSI selector can detect QSIs which can inhibit either or both the las and pqs systems [17]. The N. incisum extract had the highest inhibitory activity against the PQSI selector in 10 herbal medicines (Figures $\mathrm{S} 1$ and S2). The purity of the active compound from the $\mathrm{N}$. incisum extract was more than $98 \%$ by HPLC analysis $\left(80 \% \mathrm{MeOH} / \mathrm{H}_{2} \mathrm{O}\right)$ (Figure S3). The active compound was identified as falcarindiol by using electrospray ionization mass spectrometry, nuclear magnetic resonance spectroscopy, and HPLC analysis (Figures S4-S6, Table S4). HPLC analysis showed that the proportion of falcarindiol in the N. incisum extract was about $30 \%$. The effect of the chemical reference substance falcarindiol was detected by the PQSI selector. Falcarindiol inhibited the expression of $s a c B$ in the PQSI selector within a range of $0.5 \mu \mathrm{M}$ to $20 \mu \mathrm{M}$ (Figure $1 \mathrm{~A}$ ).

\subsection{Effect of Falcarindiol on QS-Related Virulence Phenotype}

In order to avoid imposing enormous selective pressure on bacteria, the subinhibitory concentrations of falcarindiol need to be identified. Growth curve analysis showed that the growth of PAO1 was unaffected by falcarindiol in the concentration range of $0.78125 \mu \mathrm{M}$ to $50 \mu \mathrm{M}$ (Figure 1B). The minimum inhibitory concentration (MIC) of falcarindiol to P. aeruginosa was greater than $400 \mu \mathrm{M}$ (data not shown).

Elastase contributes to tissue injury and disrupts host immune responses during P. aeruginosa infection. Elastase is encoded by lasB and regulated by LasR [18]. Pyocyanin relates to diminished lung function in cystic fibrosis $(\mathrm{CF})$ and promotes virulence due to its redox activity [19]. Rhamnolipid is a kind of biosurfactant regulated by the $r h l A / B$ operon and causes the necrosis of polymorphonuclear leukocytes. The inhibitory effect of falcarindiol on virulence factors was dose-dependent, ranging from $2.5 \mu \mathrm{M}$ to $20 \mu \mathrm{M}$. The production of elastase, pyocyanin, and rhamnolipid in PAO1 was inhibited by $37.17 \%$, $44.71 \%$, and $29.39 \%$ under $20 \mu \mathrm{M}$ falcarindiol, respectively (Figure 1C-E). The swarming motility of $P$. aeruginosa is related to the production of rhamnolipid [20]. Correspondingly, the swarming motility of $P$. aeruginosa was also inhibited by falcarindiol (data not shown).

Bacteria attach to surfaces and are embedded in a protective extracellular matrix, such as extracellular polysaccharides and proteins, to form a biofilm. The formation of 
biofilm is closely associated with QS and related to the pathogenicity of P. aeruginosa [21]. Falcarindiol reduced biofilm formation in a dose-dependent manner, which was optimal at $20 \mu \mathrm{M}$, obtaining a $50.41 \%$ inhibition (Figure $1 \mathrm{~F}$ ).
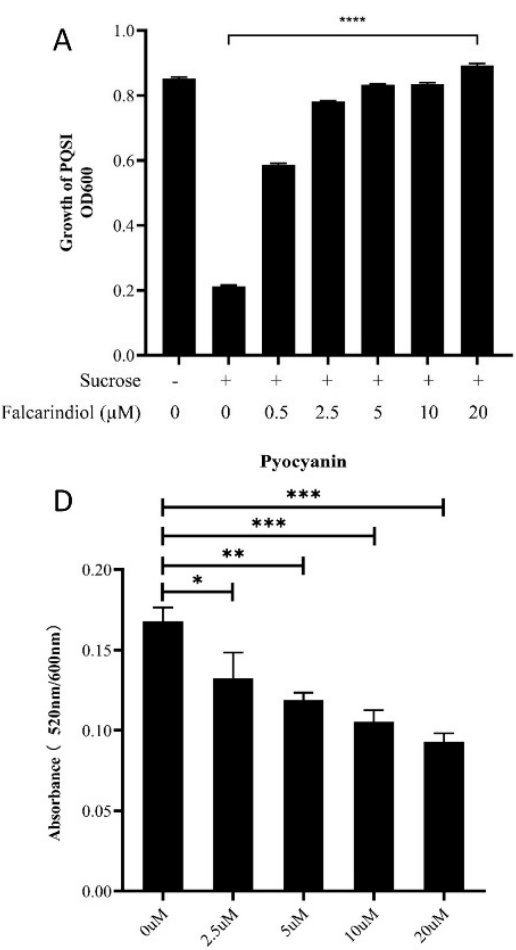

B

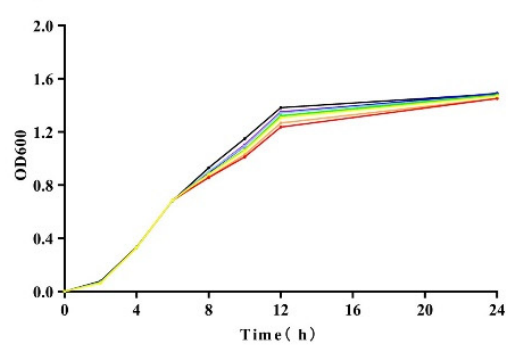

E

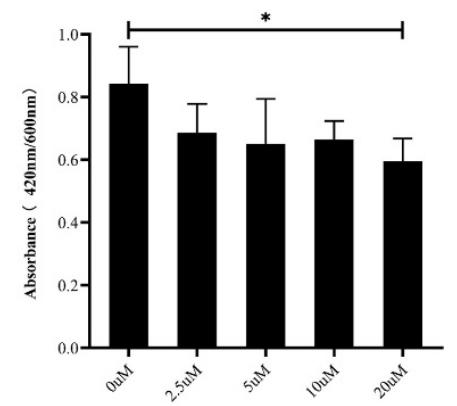

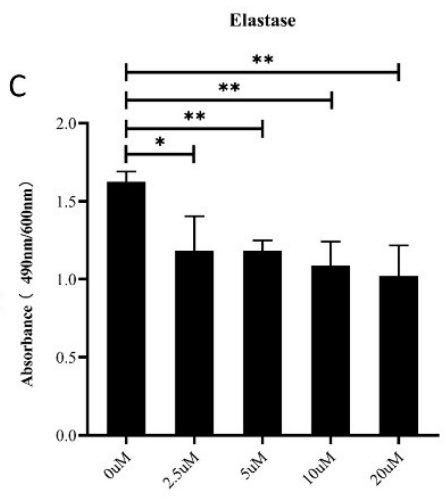

$\mathrm{F}$

Biofilm Formation

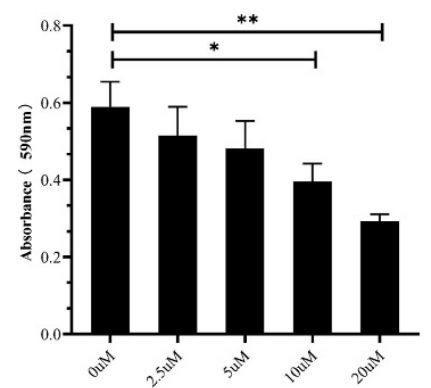

Figure 1. Effect of falcarindiol on PQSI selector (A), P. aeruginosa growth (B), elastase production (C), pyocyanin production (D), rhamnolipid production (E), and biofilm formation $(\mathbf{F})$. All experiments were performed in triplicates. The data are shown as means \pm standard errors. ${ }^{* * *} p<0.0001,{ }^{* * *} p<0.001,{ }^{* *} p<0.01,{ }^{*} p<0.05$.

\subsection{Effect of Falcarindiol on Expression of QS-Regulated Genes}

To investigate the effect of falcarindiol on the mRNA transcription of QS-related genes, las $B, r h l A, p h z H$, lasI, rhll, pqs $A$, lasR, rhlR, and $p q s R$ were selected for qRT-PCR detection. The virulence-related genes las $B, r h l A$, and $p h z H$ were regulated by $l a s, r h l$, and $p q s$ systems, respectively. After falcarindiol treatment, the mRNA expression of virulence-related genes $l a s B$, rhlA, and $p h z H$ were downregulated by $63 \%, 22.98 \%$, and $44.9 \%$, respectively. LasI, rhlI, and pqsABCD direct the synthesis of signal molecules in $P$. aeruginosa. Falcarindiol reduced the mRNA expression of lasI, rhlI, and pqsA by $29.66 \%$, $7.5 \%$, and $53.3 \%$, respectively. The mRNA expression of the QS receptor gene $r h l R$ was reduced by $44.92 \%$, that of $p q s R$ was increased by $11.86 \%$, and that of lasR was not affected by falcarindiol (Figure 2). The downregulation of $r h l I$ and $r h l A$ may be related to a decrease in the expression of $r h l R$. Because RhlR inhibits the expression of $p q s R$ and $p q s A B C D$, the upregulated expression of $p q s R$ could be attributed to a decrease of $r h l R$ expression [22]. The mRNA expression of lasI, rhll, pqs $A$, and $r h l R$ were downregulated, while the mRNA expression of their transcriptional activator LasR was not affected by falcarindiol. The downregulation of lasI, rhlI, pqsA, and rhlR may be caused by the inhibition of LasRcontrolled transcriptional activation.

\subsection{The Inhibition Effect of Falcarindiol on QS System}

The lasI and rhlI double-mutant strain P. aeruginosa QSIS-lasI containing PlasI-sacB fusion and lasR gene was unable to survive due to the presence of 3-oxo-C12-HSL and sucrose. When QSIs were added to the medium, the QSIS-lasI selector strain was rescued, and a red zone was observed around the well. The rescued effect of falcarindiol on the QSISlasI selector strain was concentration-dependent, indicating that falcarindiol inhibited the 
transcriptional activation of the lasI promoter (Figure 3A). In order to avoid the interference of the $r h l$ and pqs systems in P. aeruginosa, E. coli MG4/pKDT17 containing the lasB-lacZ fusion and las $R$ gene was used to investigate whether the transcriptional activation of LasR was inhibited by falcarindiol. E. coli pEAL08-2 and E. coli pDSY were used as the monitor strains for $p q s$ and $r h l$ systems, respectively. The inhibitory rate of falcarindiol $(50 \mu \mathrm{M})$ on $\beta$-galactosidase activity reached $38.5 \%$, indicating that falcarindiol could inhibit the transcription of LasR-controlled genes (Figure 3B). Falcarindiol also exhibited inhibitory activity in E. coli pEAL08-2 but not in E. coli pDSY (Figure 3C,D). Therefore, falcarindiol may play an inhibitory role in the las and pqs systems.

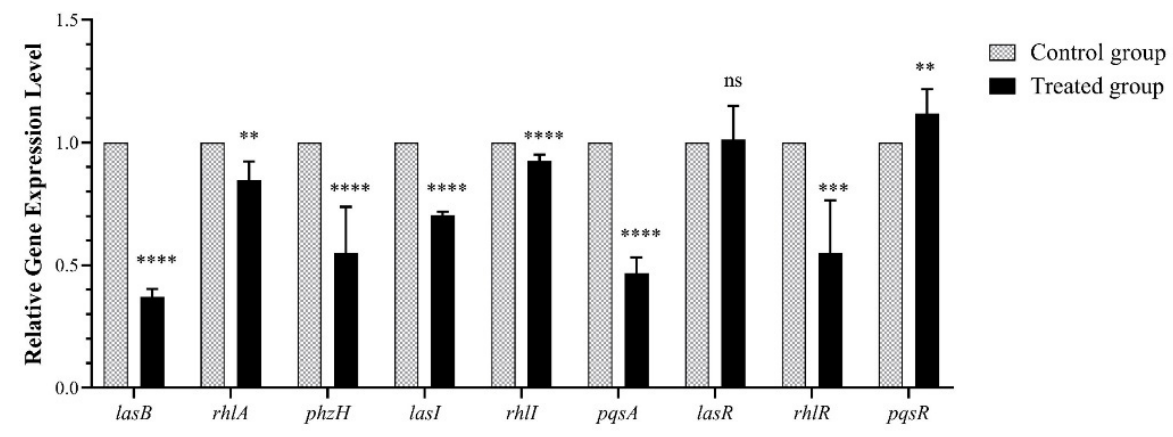

Figure 2. Effect of falcarindiol on mRNA expression. All experiments were performed in triplicates. The data are shown as means \pm standard errors. ${ }^{* * *} p<0.0001,{ }^{* * *} p<0.001,{ }^{* *} p<0.01$.

A

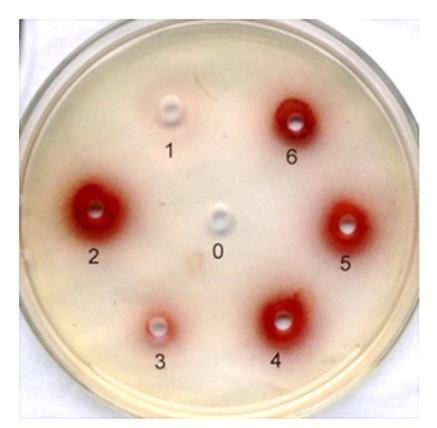

C

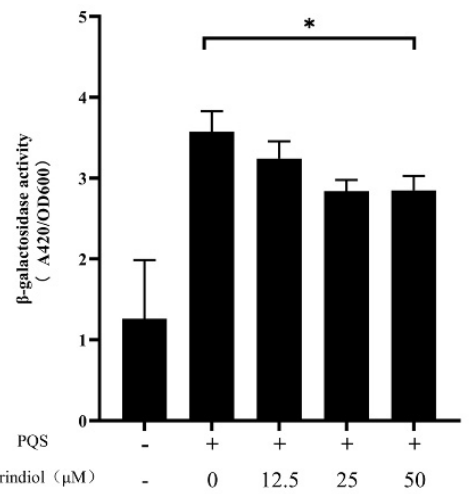

B $\quad$ MG4/PKDT17

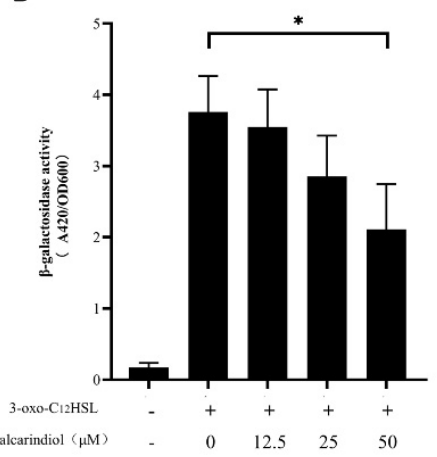

PDSY

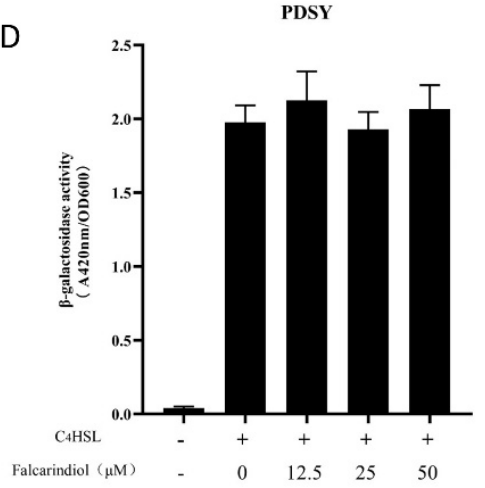

Figure 3. Effect of falcarindiol on QS inhibitory activity in P. aeruginosa QSIS-lasI (A). The negative control methanol was marked as 0 . The positive control furanone $\mathrm{C} 30(5 \mathrm{mM})$ was marked as 1 . The active ingredient $(20 \mathrm{mM})$ from the $N$. incisum extract was marked as 2 . Different concentrations of falcarindiol were marked as 3 to 6 , and the concentrations were increased sequentially $(5 \mathrm{mM}, 10 \mathrm{mM}$, $20 \mathrm{mM}, 50 \mathrm{mM}$ ). Effect of falcarindiol on las system in E. coli MG4/pKDT17 biosensor (B), pqs system in E. coli pEAL08-2 biosensor (C), and $r h l$ system in E. coli pDSY biosensor (D). All experiments were performed in triplicates. The data are shown as means \pm standard errors. ${ }^{*} p<0.05$. 


\subsection{Effect of Falcarindiol on the Binding of LasR to the Promoter Regions}

In order to detect whether falcarindiol could inhibit the binding of LasR with the promoters of downstream genes, we performed electrophoresis mobility shift assay (EMSA) experiments [23]. Falcarindiol interfered with the binding of LasR to the lasI promoter region (Figure 4). Falcarindiol inhibited the binding of LasR to the promoter of downstream genes, resulting in the inhibition of the expression of QS-regulated genes, which further affected the virulence traits of P. aeruginosa. The binding of PqsR with the pqs $A$ promoter was not affected by falcarindiol (data not shown).

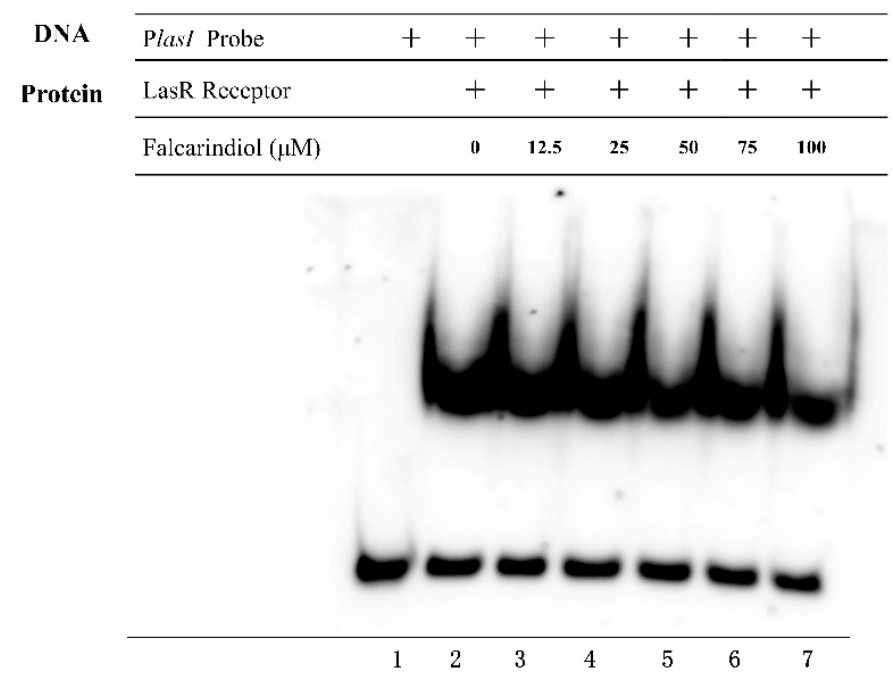

Figure 4. EMSA analysis of the effect of falcarindiol on the binding of LasR with promoter region of the lasI gene.

\subsection{Molecular Docking Analysis}

It is reported that 3-oxo-C12-HSL binds with LasR, which can then regulate the transcription of multiple downstream genes. Molecular docking was carried out to predict the binding of falcarindiol and LasR. The docking energies of LasR and falcarindiol $(-7.5 \mathrm{kcal} / \mathrm{mol})$ were similar to those of natural ligand and LasR $(-8.0 \mathrm{kcal} / \mathrm{mol})$ (Table S5). The natural ligand 3-oxo-C12-HSL was able to form five hydrogen bonds with amino acid residues (Tyr56, Trp60, Asp73, Thr75, Ser129) (Figure 5A,B). Falcarindiol was able to form three hydrogen bonds (Tyr47, Thr75, Ser129) (Figure 5C,D). In a manner similar to QSI butein, falcarindiol could interact with the Tyr47 residue and disrupt the stability of LasR [24]. The instability of LasR may inhibit binding with the promoter region of the target genes, in turn reducing its transcriptional activation. 

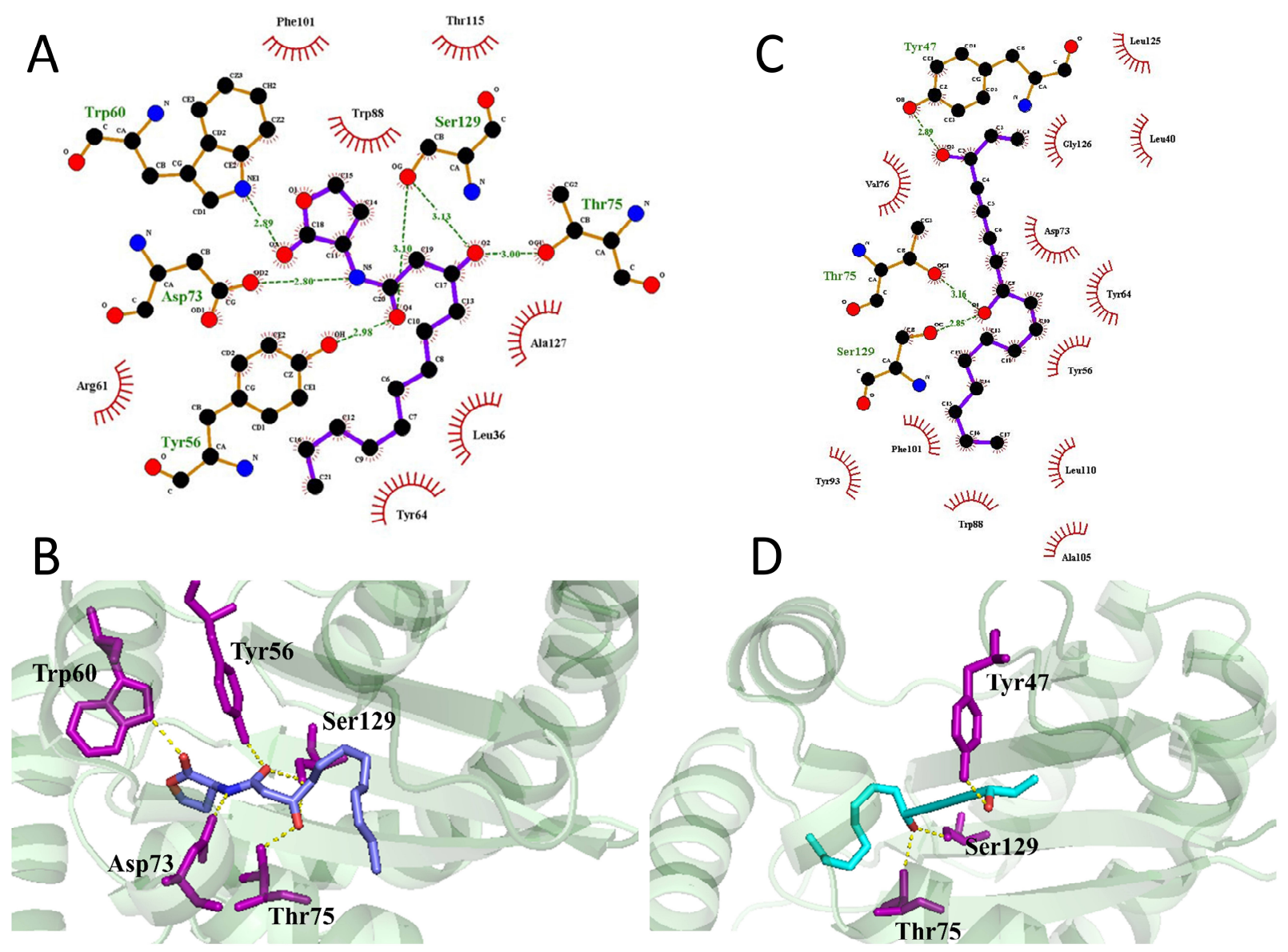

Figure 5. The 2D (A) and 3D (B) interaction maps of 3-oxo-C12-HSL and LasR. The 2D (C) and 3D (D) interaction maps of falcarindiol and LasR.

\section{Discussion}

P. aeruginosa can easily acquire resistance to various antibiotics. QSIs can inhibit $P$. aeruginosa virulence without affecting bacterial growth, which imposes less selection pressure for bacterial resistance. Falcarindiol was identified from N. incisum and exhibited QS inhibitory activity. Our research provided a reference for the purification of the natural polyacetylenic compound. The research on the biological activity of falcarindiol provided a theoretical basis for the development and application of new antibiotics. Previous studies showed that falcarindiol had a variety of pharmacological activities, such as anti-apoptotic, antioxidant, and anti-inflammatory [25]. Falcarindiol also has anti-bacterial activity [26]. A study by Zhang et al. indicated that falcarindiol can inhibit the activation of lasI, rhll, and lasB promoters in the $p$-lux promoter-reporter system [27]. However, the molecular mechanisms underlying the falcarindiol-mediated inhibition of QS remain unclear.

Using the P. aeruginosa QSIS-lasI, E. coli MG4/pKDT17, E. coli pEAL08-2, and E. coli pDSY assay, we found that falcarindiol could inhibit the las and pqs systems. Falcarindiol reduced the mRNA expression levels of lasI, rhlI, pqsA, and $r h l R$, which were activated by the transcriptional activator LasR. However, the mRNA expression of las $R$ was not affected by falcarindiol. EMSA results confirmed that falcarindiol inhibited the DNA-binding capability of LasR. Falcarindiol interfered with the transcriptional activity of LasR, resulting in a decreased expression of LasR-controlled downstream genes (lasB, rhlA, phzH, lasI, rhlI, $p q s A$, and $r h l R$ ). The inhibitory effect of falcarindiol on the $p q s$ system may be controlled by other mechanisms. LasR bonded with 3-oxo-C12-HSL to activate transcription. Molecular docking results revealed that falcarindiol was able to form hydrogen bonds with Tyr47, destabilizing the conformation of LasR [28]. The binding of Tyr47 and ligands results in the ligand being unable to form hydrogen bonds with Trp60 and Asp73 residues [24]. 
Falcarindiol interfered with the binding of natural ligands with LasR. Meanwhile, the LasR-mediated transcriptional activation of downstream gene promoters was inhibited by falcarindiol in P. aeruginosa QSIS-lasI and E. coli MG4/pKDT17. Falcarindiol exhibited weak inhibitory activity against the AHL-mediated QS system in Chromobacterium violaceum (data not shown). Because of the long alkyl chain in its structure, falcarindiol showed a stronger inhibition effect on the las system than on the C6-HSL-mediated QS system. Therefore, falcarindiol was expected to be an inhibitor of the long-chain AHL-mediated QS system.

\section{Materials and Methods}

\subsection{Bacterial Strains, Plasmids, and Materials}

The bacterial strains and plasmids used in our research are listed in Table S1. The primers for PCR and real time RT-PCR are listed in Tables S2 and S3, respectively. The chemical reference substance falcarindiol was purchased from a Chengdu PurechemStandard Co. (Chengdu, China), LTD, and was used to evaluate anti-QS activity against P. aeruginosa PAO1.

\subsection{Screening of QSIs}

The promoter region of $p q s A$ was amplified and ligated into the SphI and XbaI sites of plasmid PlasB-sacB1. The resultant recombinant plasmid PpqsA-sacB was transformed into $P$. aeruginosa $\mathrm{PAO} 1$ to construct the PQSI strain. The overnight culture of the PQSI strain was diluted 1:1000 in LB with $80 \mu \mathrm{g} / \mathrm{mL}$ gentamicin and 1.5\% sucrose. Crude extracts $(50 \mathrm{mg} / \mathrm{mL})$ of Chinese herbal medicines were dissolved in methanol and added to the culture. Bacterial growth was determined at $600 \mathrm{~nm}$ after incubation for $8 \mathrm{~h}$ at $37^{\circ} \mathrm{C}$.

\subsection{Purification of Active Compound}

$N$. incisum powder was soaked in ethyl acetate and extracted by ultrasonic waves. After the filtrate was evaporated and dried, the extract was dissolved with methanol. The methanol-soluble extract of $N$. incisum was fractionated via a silica gel gradient vacuum liquid chromatography column eluting with petroleum ether and a step gradient of $\mathrm{CH}_{2} \mathrm{Cl}_{2} / \mathrm{MeOH}(v / v$ 100:0, 100:1, 50:1, 20:1, and 0:100) to give 6 fractions, based on TLC analysis. Fraction 4 obtained from $\mathrm{CH}_{2} \mathrm{Cl}_{2} / \mathrm{MeOH}(v / v=50: 1)$ was subjected to Sephadex LH-20 chromatography with methanol to afford 29 subfractions (S1-S29). Subfractions were analyzed by TLC, and 3 subfractions (S18-S20) had anti-QS activity. Subfraction S20 was further purified into four constituents by preparative high-performance liquid chromatography (HPLC) (Shimadzu, Kyodo, Japan) $\left(70 \% \mathrm{MeOH} / \mathrm{H}_{2} \mathrm{O}\right)$. The anti-QS activities of the four constituents were determined by the PQSI selector.

\subsection{Growth Curve Assay}

The overnight culture of $P$. aeruginosa PAO1 was diluted to an OD600 of 0.01 with fresh LB medium and incubated with falcarindiol $(0-50 \mu \mathrm{M})$ at $37^{\circ} \mathrm{C}$. Bacterial growth was observed by measuring $\mathrm{OD}$ at $600 \mathrm{~nm}$.

\subsection{Agar Well-Diffusion Assay}

The P. aeruginosa QSIS-lasI biosensor was used to detect the QS inhibitory effects of falcarindiol [29]. Falcarindiol and C30 were dissolved in methanol and added into wells made in LB agar medium mixed with an overnight culture of P. aeruginosa QSIS-lasI, sucrose, $0.75 \mathrm{mg} / \mathrm{mL}$ 2,3,5-triphenyltetrazolium chloride (TTC), and $60 \mathrm{nM}$ 3-oxo-C12-HSL. The red zones around the wells were observed after incubating at $37^{\circ} \mathrm{C}$ overnight.

\subsection{Analysis of lasB, pqsA, and rhlA Transcriptional Activity in E. coli}

The detection of $\beta$-galactosidase activity in E. coli MG4/pKDT17, E. coli pEAL082 [30,31], and E. coli pDSY was described previously. The overnight culture of E. coli MG4/pKDT17, E. coli pEAL08-2, and E. coli pDSY was diluted 1:100 in LB and then 
incubated with falcarindiol at $37^{\circ} \mathrm{C}$ for $8 \mathrm{~h}$. A $\beta$-galactosidase Assay Kit (Beyotime, Shanghai, China) was used to quantify $\beta$-galactosidase activities.

\subsection{Virulence Factor Assays}

The overnight culture of P. aeruginosa PAO1 was diluted 1:100 with fresh LB and then incubated with falcarindiol at $37^{\circ} \mathrm{C}$. After incubation for $6 \mathrm{~h}$, the supernatant was collected for the quantification of virulence factors. Pyocyanin was extracted from $800 \mu \mathrm{L}$ supernatant with $600 \mu \mathrm{L}$ chloroform. The $500 \mu \mathrm{L}$ organic layer was incubated with $200 \mu \mathrm{L}$ $\mathrm{HCl}(0.2 \mathrm{~N})$ at $37^{\circ} \mathrm{C}$ for $30 \mathrm{~min}$. A $150 \mu \mathrm{L}$ aliquot of the water layer was removed and its OD520 was measured [32]. Elastase activity was measured through elastin Congo red assays. The $800 \mu \mathrm{L}$ elastin Congo red solution $(3 \mathrm{mg} / \mathrm{mL}$ in $0.1 \mathrm{M}$ Tris- $\mathrm{HCl} \mathrm{pH}=7.2$; $1 \mathrm{mM} \mathrm{CaCl}_{2}$ ) was incubated with $400 \mu \mathrm{L}$ cell-free supernatant at $37^{\circ} \mathrm{C}$ for $6 \mathrm{~h}$. The samples were centrifuged and measured at $490 \mathrm{~nm}$ [33]. The rhamnolipid was measured by an orcinol assay [34]. An $800 \mu \mathrm{L}$ aliquot of supernatant was extracted with $1 \mathrm{~mL}$ diethyl ether. The organic phase $(800 \mu \mathrm{L})$ was evaporated, and the residue was resuspended in a solution (100 $\mu \mathrm{L}$ sterile water, $700 \mu \mathrm{L} 70 \% \mathrm{H}_{2} \mathrm{SO}_{4}$, and $100 \mu \mathrm{L} \mathrm{1.6 \%} \mathrm{orcinol).} \mathrm{The} \mathrm{solution}$ was measured at $420 \mathrm{~nm}$ after incubating at $80^{\circ} \mathrm{C}$ for $30 \mathrm{~min}$.

\subsection{Biofilm Formation Assay}

The overnight culture of P. aeruginosa PAO1 was diluted 1:10,000 with fresh LB and transferred into a $1.5 \mathrm{~mL}$ centrifuge tube with falcarindiol at $2.5 \mu \mathrm{M}$ to $20 \mu \mathrm{M}$. Non-adherent cells were eliminated by PBS after static incubation for $12 \mathrm{~h}$. The biofilm was stained with $0.1 \%$ crystal violet for $15 \mathrm{~min}$. The stained biofilm was dissolved with $33 \%(v / v)$ glacial acetic acid and measured at OD590 $\mathrm{nm}$.

\subsection{Real-Time RT-PCR Assay}

The overnight $P$. aeruginosa PAO1 culture was diluted 1:100 and then incubated with falcarindiol at $50 \mu \mathrm{M}$ for another $6 \mathrm{~h}$. Total RNA was isolated with an RNA isolation kit (Nobelab Biotechnologies, Beijing, China) [35]. HiScript III RT Super Mix (Vazyme Biotech, Nanjing, China) and ChamQ Universal SYBR qPCR Master Mix (Vazyme Biotech, Nanjing, China) were used for reverse transcription and amplification, respectively. The reference gene $r p s l$ was used to normalize qRT-PCR. The comparative threshold method $\left(2^{-\Delta \Delta \mathrm{Ct}}\right)$ was used to calculate the relative expression of the target genes.

\subsection{EMSA}

The recombinant expression vector pET28a-LasR (for LasR production) was transformed into E. coli BL21 (DE3). The transformants were incubated with $1 \mathrm{mM}$ IPTG and $100 \mu \mathrm{M}$ 3-oxo-C12-HSL at $18{ }^{\circ} \mathrm{C}$ for $16 \mathrm{~h}$. Whole-cell lysates containing the LasR protein were collected and used in the EMSA [36]. The promoter of the lasI gene was amplified from the genome of P. aeruginosa and used as a probe. Biotin-labeled PlasI probe was prepared by the EMSA probe labeling kit (Beyotime, Shanghai, China). Different concentrations of falcarindiol $(0 \mu \mathrm{M}, 12.5 \mu \mathrm{M}, 25 \mu \mathrm{M}, 50 \mu \mathrm{M}, 75 \mu \mathrm{M}$, and $100 \mu \mathrm{M}$, dissolved in DMSO) were added to the reaction mix. The binding reactions for LasR-PlasI interaction and gel-shift assays were performed with the EMSA/Gel-Shift Kit (Beyotime, Shanghai, China).

\subsection{Molecular Docking Analysis}

Molecular docking was performed as previously reported [24]. The structure of the receptor protein LasR (PDB ID, 3IX3) was downloaded from the Protein Data Bank (PDB). The molecular docking study was performed by Autodock 4.2.6 (The Scripps Research Institute, Jolla, LA USA). The conformations and interactions between falcarindiol and LasR were visualized by PyMol 2.3.0 (Schrodinger, New York, NY, USA). and LigPlot+ v.1.4 (EMBL-EBI, Cambridge, UK). 


\section{Conclusions}

Our results indicate that falcarindiol identified from N. incisum can inhibit the QS of $P$. aeruginosa and exert a significant inhibitory effect on the formation of biofilm and the production of virulence factors (elastase, pyocyanin, and rhamnolipid). The mRNA expression of QS-related genes (lasB, phzH, rhlA, lasI, rhlI, pqsA, and rhlR) was downregulated by falcarindiol. Falcarindiol inhibited the binding of the transcription factor LasR with the lasI promoter region and the transcriptional activation of downstream genes. Falcarindiol has great potential to be developed as an anti-infective agent. The therapeutic effects of falcarindiol on P. aeruginosa infection need to be further studied.

Supplementary Materials: The following are available online. Table S1: Bacterial strains and plasmids. Table S2: The primer sequences for cloning. Table S3: The primer sequences for real-time RT-PCR. Table S4: NMR spectroscopy of the active compound purified from the Notopterygium incisum extract. Table S5: Details of the docked complex of LasR with 3-oxo-C12-HSL and falcarindiol. Figure S1: The inhibitory activity of Chinese herbal medicines against PQSI selector. Figure S2: The inhibitory activity of Notopterygium incisum extract against PQSI selector. Figure S3: HPLC analysis of the active compound purified from the Notopterygium incisum extract. Figure S4: ${ }^{1} \mathrm{H}-\mathrm{NMR}$ spectroscopy of the active compound purified from the Notopterygium incisum extract (Solvent: DMSOd6). Figure S5: ${ }^{13} \mathrm{C}-\mathrm{NMR}$ spectroscopy of the active compound purified from the Notopterygium incisum extract (Solvent: DMSO-d6). Figure S6: Comparison of HPLC retention times of the active compound purified from the Notopterygium incisum extract (A), the chemical reference substance of falcarindiol (B), and their mixture (C).

Author Contributions: C.Z. performed the experiments (including EMSA and anti-QS activity evaluation in vitro), data analysis, and prepared the paper. H.Z. carried out the screening and purification of falcarindiol and the anti-QS activity evaluation in vitro. L.Z. (Liman Zhou) contributed to the purification of falcarindiol. H.J. was in charge of real-time RT-PCR. L.Z. (Lu Zhao) was responsible for virulence factors assays. W.Y. initiated the project. Q.G. conceived, designed the work, and revised the paper. All authors have read and agreed to the published version of the manuscript.

Funding: This research was supported by the National Natural Science Foundation of China (No. 81102368), the Qingdao Scientific and Technological Innovation center for Marine Biomedicine Development Grant (2017-CXZX01-3-8), and the Key R\&D Program of Shandong Province (2018GHY115047).

Acknowledgments: We would like to thank E. Peter Greenberg and Robert J.C. McLean for providing us with the strains.

Conflicts of Interest: The authors declare no conflict of interest.

Sample Availability: Samples of the compounds are available from the authors.

\section{References}

1. Sadikot, R.T.; Blackwell, T.S.; Christman, J.W.; Prince, A.S. Pathogen-host interactions in Pseudomonas aeruginosa pneumonia. Am. J. Respir. Crit Care Med. 2005, 171, 1209-1223. [CrossRef]

2. Lopez-Causape, C.; Cabot, G.; Del Barrio-Tofino, E.; Oliver, A. The versatile mutational resistome of Pseudomonas aeruginosa. Front. Microbiol. 2018, 9, 685. [CrossRef] [PubMed]

3. Fuqua, W.C.; Winans, S.C.; Greenberg, E.P. Quorum sensing in bacteria: The LuxR-LuxI family of cell density-responsive transcriptional regulators. J. Bacteriol. 1994, 176, 269-275. [CrossRef] [PubMed]

4. Dong, Y.H.; Wang, L.H.; Xu, J.L.; Zhang, H.B.; Zhang, X.F.; Zhang, L.H. Quenching quorum-sensing-dependent bacterial infection by an $N$-acyl homoserine lactonase. Nature 2001, 411, 813-817. [CrossRef]

5. Soukarieh, F.; Williams, P.; Stocks, M.J.; Camara, M. Pseudomonas aeruginosa quorum sensing systems as drug discovery targets: Current position and future perspectives. J. Med. Chem. 2018, 61, 10385-10402. [CrossRef]

6. Wagner, V.E.; Iglewski, B.H. P. aeruginosa biofilms in CF infection. Clin. Rev. Allergy Immunol. 2008, 35, 124-134. [CrossRef]

7. Schuster, M.; Greenberg, E.P. A network of networks: Quorum-sensing gene regulation in Pseudomonas aeruginosa. Int. J. Med. Microbiol. 2006, 296, 73-81. [CrossRef]

8. Kostylev, M.; Kim, D.Y.; Smalley, N.E.; Salukhe, I.; Greenberg, E.P.; Dandekar, A.A. Evolution of the Pseudomonas aeruginosa quorum-sensing hierarchy. Proc. Natl. Acad. Sci. USA 2019, 116, 7027-7032. [CrossRef]

9. Schuster, M.; Greenberg, E.P. Early activation of quorum sensing in Pseudomonas aeruginosa reveals the architecture of a complex regulon. BMC Genom. 2007, 8, 287. [CrossRef] 
10. Gambello, M.J.; Kaye, S.; Iglewski, B.H. LasR of Pseudomonas aeruginosa is a transcriptional activator of the alkaline protease gene (apr) and an enhancer of exotoxin a expression. Infect. Immun. 1993, 61, 1180-1184. [CrossRef]

11. Diggle, S.P.; Crusz, S.A.; Camara, M. Quorum sensing. Curr. Biol. 2007, 17, R907-R910. [CrossRef]

12. Emam, M.; Abdel-Haleem, D.R.; Salem, M.M.; Abdel-Hafez, L.J.M.; Latif, R.R.A.; Farag, S.M.; Sobeh, M.; El Raey, M.A. Phytochemical Profiling of lavandula coronopifolia poir. aerial parts extract and its larvicidal, antibacterial, and antibiofilm activity against Pseudomonas aeruginosa. Molecules 2021, 26, 1710. [CrossRef]

13. Mostafa, I.; Abbas, H.A.; Ashour, M.L.; Yasri, A.; El-Shazly, A.M.; Wink, M.; Sobeh, M. Polyphenols from salix tetrasperma impair virulence and inhibit quorum sensing of Pseudomonas aeruginosa. Molecules 2020, 25, 1341. [CrossRef]

14. Luo, J.; Kong, J.L.; Dong, B.Y.; Huang, H.; Wang, K.; Wu, L.H.; Hou, C.C.; Liang, Y.; Li, B.; Chen, Y.Q. Baicalein attenuates the quorum sensing-controlled virulence factors of Pseudomonas aeruginosa and relieves the inflammatory response in $P$. aeruginosainfected macrophages by downregulating the MAPK and NFkappaB signal-transduction pathways. Drug Des. Dev. Ther. 2016, 10, 183-203. [CrossRef]

15. Kumar, L.; Chhibber, S.; Kumar, R.; Kumar, M.; Harjai, K. Zingerone silences quorum sensing and attenuates virulence of Pseudomonas aeruginosa. Fitoterapia 2015, 102, 84-95. [CrossRef]

16. Hernando-Amado, S.; Alcalde-Rico, M.; Gil-Gil, T.; Valverde, J.R.; Martinez, J.L. Naringenin inhibition of the Pseudomonas aeruginosa quorum sensing response is based on its time-dependent competition with $\mathrm{N}$-(3-Oxo-dodecanoyl)-L-homoserine lactone for LasR binding. Front. Mol. Biosci. 2020, 7, 25. [CrossRef]

17. McGrath, S.; Wade, D.S.; Pesci, E.C. Dueling quorum sensing systems in Pseudomonas aeruginosa control the production of the Pseudomonas quinolone signal (PQS). FEMS Microbiol. Lett. 2004, 230, 27-34. [CrossRef]

18. Saint-Criq, V.; Villeret, B.; Bastaert, F.; Kheir, S.; Hatton, A.; Cazes, A.; Xing, Z.; Sermet-Gaudelus, I.; Garcia-Verdugo, I.; Edelman, A.; et al. Pseudomonas aeruginosa LasB protease impairs innate immunity in mice and humans by targeting a lung epithelial cystic fibrosis transmembrane regulator-IL-6-antimicrobial-repair pathway. Thorax 2018, 73, 49-61. [CrossRef]

19. Xiao, G.; Deziel, E.; He, J.; Lepine, F.; Lesic, B.; Castonguay, M.H.; Milot, S.; Tampakaki, A.P.; Stachel, S.E.; Rahme, L.G. MvfR, a key Pseudomonas aeruginosa pathogenicity LTTR-class regulatory protein, has dual ligands. Mol. Microbiol. 2006, 62, 1689-1699. [CrossRef]

20. Davey, M.E.; Caiazza, N.C.; O'Toole, G.A. Rhamnolipid surfactant production affects biofilm architecture in Pseudomonas aeruginosa PAO1. J. Bacteriol. 2003, 185, 1027-1036. [CrossRef]

21. Brackman, G.; Coenye, T. Quorum sensing inhibitors as anti-biofilm agents. Curr. Pharm. Des. 2015, 21, 5-11. [CrossRef]

22. Papenfort, K.; Bassler, B.L. Quorum sensing signal-response systems in gram-negative bacteria. Nat. Rev. Microbiol. 2016, 14, 576-588. [CrossRef]

23. Revzin, A.; Ceglarek, J.A.; Garner, M.M. Comparison of nucleic acid-protein interactions in solution and in polyacrylamide gels. Anal. Biochem. 1986, 153, 172-177. [CrossRef]

24. Zhong, L.; Ravichandran, V.; Zhang, N.; Wang, H.; Bian, X.; Zhang, Y.; Li, A. Attenuation of Pseudomonas aeruginosa quorum sensing by natural products: Virtual screening, evaluation and biomolecular interactions. Int. J. Mol. Sci. 2020, 21, 2190. [CrossRef]

25. Kramer, M.; Muhleis, A.; Conrad, J.; Leitenberger, M.; Beifuss, U.; Carle, R.; Kammerer, D.R. Quantification of polyacetylenes in apiaceous plants by high-performance liquid chromatography coupled with diode array detection. Z. Nat. C J. Biosci. 2011, 66, 319-327. [CrossRef]

26. Tan, K.W.; Killeen, D.P.; Li, Y.; Paxton, J.W.; Birch, N.P.; Scheepens, A. Dietary polyacetylenes of the falcarinol type are inhibitors of breast cancer resistance protein (BCRP/ABCG2). Eur. J. Pharmacol. 2014, 723, 346-352. [CrossRef]

27. Zhang, P.; Wu, Q.; Chen, L.; Duan, K. Virulence-inhibiting herbal compound falcarindiol significantly reduced mortality in mice infected with Pseudomonas aeruginosa. Antibiotics 2020, 9, 136. [CrossRef]

28. O'Reilly, M.C.; Dong, S.H.; Rossi, F.M.; Karlen, K.M.; Kumar, R.S.; Nair, S.K.; Blackwell, H.E. Structural and biochemical studies of non-native agonists of the lasr quorum-sensing receptor reveal an L3 loop "out" conformation for LasR. Cell Chem. Biol. 2018, 25, 1128-1139.e1123. [CrossRef] [PubMed]

29. Wang, L.; Zou, S.; Yin, S.; Liu, H.; Yu, W.; Gong, Q. Construction of an effective screening system for detection of Pseudomonas aeruginosa quorum sensing inhibitors and its application in bioautographic thin-layer chromatography. Biotechnol. Lett. 2011, 33, 1381-1387. [CrossRef] [PubMed]

30. Pearson, J.P.; Gray, K.M.; Passador, L.; Tucker, K.D.; Eberhard, A.; Iglewski, B.H.; Greenberg, E.P. Structure of the autoinducer required for expression of Pseudomonas aeruginosa virulence genes. Proc. Natl. Acad. Sci. USA 1994, 91, 197-201. [CrossRef] [PubMed]

31. Cugini, C.; Calfee, M.W.; Farrow, J.M., 3rd; Morales, D.K.; Pesci, E.C.; Hogan, D.A. Farnesol, a common sesquiterpene, inhibits PQS production in Pseudomonas aeruginosa. Mol. Microbiol. 2007, 65, 896-906. [CrossRef] [PubMed]

32. Muh, U.; Schuster, M.; Heim, R.; Singh, A.; Olson, E.R.; Greenberg, E.P. Novel Pseudomonas aeruginosa quorum-sensing inhibitors identified in an ultra-high-throughput screen. Antimicrob. Agents Chemother. 2006, 50, 3674-3679. [CrossRef] [PubMed]

33. Sun, S.; Dai, X.; Sun, J.; Bu, X.; Weng, C.; Li, H.; Zhu, H. A diketopiperazine factor from Rheinheimera aquimaris QSI02 exhibits anti-quorum sensing activity. Sci. Rep. 2016, 6, 39637. [CrossRef] [PubMed] 
34. Pearson, J.P.; Pesci, E.C.; Iglewski, B.H. Roles of Pseudomonas aeruginosa las and rhl quorum-sensing systems in control of elastase and rhamnolipid biosynthesis genes. J. Bacteriol. 1997, 179, 5756-5767. [CrossRef] [PubMed]

35. Zhang, M.; Wang, M.; Zhu, X.; Yu, W.; Gong, Q. Equisetin as potential quorum sensing inhibitor of Pseudomonas aeruginosa. Biotechnol. Lett. 2018, 40, 865-870. [CrossRef]

36. Paczkowski, J.E.; Mukherjee, S.; McCready, A.R.; Cong, J.P.; Aquino, C.J.; Kim, H.; Henke, B.R.; Smith, C.D.; Bassler, B.L. Flavonoids suppress Pseudomonas aeruginosa virulence through allosteric inhibition of quorum-sensing receptors. J. Biol. Chem. 2017, 292, 4064-4076. [CrossRef] 